\title{
MOLECULAR TAXONOMY OF YEASTS
}

\author{
T. DEÁK \\ Department of Microbiology and Biotechnology, University of Horticulture and Food Science, \\ Budapest, Hungary
}

\begin{abstract}
In the last two decades the application of molecular techniques has had a major impact on the classification of yeasts. The nuclear DNA relatedness has become the basis of species delineation. Molecular fingerprinting methods such as analysis of restriction fragment length polymorphisms, random amplified polymorphic DNA, PCR-amplified sequences and fragments, pulsed field gel electrophoresis of chromosome DNA and others allow intraspecies differentiation and typing. The most far reaching method has been the sequencing of various parts of ribosomal DNA that has made for the first time possible to assess the phylogenetic relationships among yeasts at different taxonomic levels. Based on the molecular data obtained so far several changes have been introduced in the classification of yeasts, however, substantial restructuring of current taxonomic schemes with the consequence of numerous nomenclatural changes must await further studies.
\end{abstract}

\section{Introduction}

Molecular techniques are increasingly used in the taxonomic study of yeasts. Historically, the DNA base composition $(\mathrm{G}+\mathrm{C}$ mol \%) was first used for species definition, later, however, DNA relatedness (DNA/DNA homology, nuclear DNA hybridization) has become the standard basis to delineate species. Comparisons of ribosomal RNA and its gene (rDNA) have made the largest impact on yeast taxonomy, as the variable and conservative sequences of rRNA genes allow to assess both close and distant relationships at various taxonomic levels. Moreover, these studies have also led to establish phylogenetic and evolutionary relationships among yeasts and their connections with other fungi [1]. Various other molecular methods, such as analysis of restriction fragment length polymorphisms (RFLP), pulsed field gel electrophoresis of chromosomal DNA (PFGE), random amplified polymorphic DNA (RAPD), as well as PCR-based techniques have been used extensively for typing species, because their sensitivity reveals intraspecific relations. Some of these and other molecular techniques (e.g. species-specific DNA-probes and sequencing of specific rDNA regions) can be used for yeast identification [2].

TIBOR DEÁK

Department of Microbiology and Biotechnology, University of Horticulture and Food Science

Somlói út 14-16, H-1118 Budapest, Hungary 
In this review the impact of the use of molecular techniques on yeast taxonomy will be discussed at decreasing levels of taxons. First, the place of yeasts in the kingdom of fungi and among the fungal divisions will be outlined, followed by the overview of higher taxa of yeasts (orders and families). Examples will be given for the recent classification of genera and species, and for the typing of strains at intraspecies level.

\section{Place of yeasts among fungi}

Based on the evidence from $5 \mathrm{~S}$ and $18 \mathrm{~S}$ rDNA sequences it is now a consensus in the taxonomy of fungi that the true fungi comprise four monophyletic groups: chytridiomycetes, zygomycetes, ascomycetes and basidiomycetes. Yeasts belong to the latter two groups (the so-called higher fungi), and in contrast to the majority of these organisms, which are hyphal and have chitinous cell walls, the yeasts are traditionally defined as unicellular organisms with mannan and glucan in the cell wall. In addition, yeasts are usually considered as fermentative organisms that vegetatively reproduce themselves by budding. This definition of yeasts is clearly imprecise, and the name "yeasts" can be used only as a practical term. The borderline between filamentous and unicellular, budding forms is not sharp as many fungi, both ascomycetes and basidiomycetes, may be dimorphic and have either hyphal or yeast-like growth phases. Traditionally, those fungi are considered yeasts that exist primarily in unicellular state, although a number of them can also produce hyphae. The number of described species in the successive taxonomic treatises on yeasts has steadily increased as more fungi with budding states are accepted as yeasts, which currently have 100 genera and over 700 species [3].

Phylogenetic analysis of rDNA sequences have revealed three major evolutionary lineages in both ascomycetes and basidiomycetes, and yeasts or yeast-like organisms are found in each of these groups [4, 5].

The Ascomycota is composed of three classes: the Archiascomycetes, the Hemiascomycetes and the Euascomycetes. The Archiascomycetes (basal or early ascomycetes) diverged prior the separation of the other two major ascomycetous lineages [6]. They include diverse taxa among them the fission yeasts. One of the most striking result of molecular studies has been the demonstration of the deep phylogenetic distance between the fission yeast (genus Schizosaccharomyces) and the budding yeasts (Saccharomyces and similar genera).

The ascomycetous true yeasts have traditionally been grouped in Hemiascomycetes, that also included the taphrinas. The latters having transferred to Archiascomycetes, the Hemiascomycetes now becomes limited to the single order, Endomycetales. However, the identity of the type species of the genus Endomyces is doubtful, and recently the ascomycetous yeasts are classified in the order Saccharomycetales with the type genus Saccharomyces. Several yeast-like forms occur in cultures of filamentous ascomycetes fungi (Euascomycetes). These, among them the socalled black yeasts (e.g. Aureobasidium) are, however, not considered as yeasts. The main 
distinctive character between hemiascomycete yeasts and euascomycete fungi is the formation of fruiting bodies in the teleomorphic states of the latters, although their anamorphs may be confused with yeasts.

Molecular analysis of basidiomycetous fungi has resulted in conflicting results with the traditional classification based mainly on basidial morphology. It has been demonstrated that higher taxa characterized with the formation of phragmobasidia or holobasidia or teliospores do not represent natural phylogenetic groups. Evidence from the $18 \mathrm{~S}$ rDNA sequences for the three main classes of basidiomycetes is rather corroborated with ultrastructural and chemotaxonomic features, such as septal pore, spindle pole body or cellular carbohydrate composition [5]. Accordingly, Urediniomycetes, the rusts, are characterized with simple septal pore and fucose or rhamnose in cell walls, Ustilaginomycetes, the smuts, may also possess simple pores but no pentoses occur in the cellular carbohydrates, whereas the third group, Hymenomycetes are characterized with complex septa and xylose in the cell wall. Basidiomycetous yeasts are distributed in all three phylogenetic lineages and can be integrated with filamentous forms. However, the earlier criteria used for the classification of basidiomycetous yeasts, such as the formation of teliospores or ballistoconidia do not correspond with the phylogenetic grouping. Yeasts with teliospores and/or ballistoconidia can be found in all three classes, however, in Hymenomycetes they are confined only to the subgroup of tremellaceous fungi.

In the traditional classification of fungi, species lacking sexual reproduction were assembled in the form-taxon Deuteromycota, and anamorphic yeast forms were placed in Blastomycetes. The current trend is to combine anamorphs with teleomorphs (mitosporic forms with meiosporic ones) into one classification [7]. This meets great difficulties with filamentous fungi possessing several different anamorphs corresponding to a given teleomorph, and vice versa. With yeasts, the conditions are more favourable, and by the concerted use of morphological, chemical and molecular data, a unified phylogenetic classification can be attained.

\section{Higher taxa of yeasts}

In the framework of fungal classes outlined above, yeasts can be placed in several orders and families. Limited sequence data make, at present, their circumscription uncertain and the classification at these levels is far from complete [8].

Members of the Archiascomycetes appear to be in rather distant phylogenetic relationships, hence they are differentiated at ordinal level. One of the four orders is Schizosaccharomycetales, which can be contrasted with the single order Saccharomycetales (synonym Endomycetales) comprising all ascomycetous yeasts and yeast-like organisms (Hemiascomycetes). The inclusion among these the hyphal yeasts, families of Endomycetaceae, Dipodascaceae, Saccharomycopsidaceae, Ascoideaceae, Cephaloascaceae and Eremotheciaiceae is well supported by molecular data [9]. An interesting case is the last family, the members of which are characterized by long, needleshape spores, in common with the species of Metschnikowia. Hence they were formerly included in one family, Metschnikowiaceae. Contrary to the apparent morphological 
similarity, they form distinct phylogenetic lineages representing separate families. A similar case may be the family Saccharomycodaceae, in which the bipolar budding is a shared morphological character of the genera included. Nevertheless, they fall on different branches of the $18 \mathrm{~S}$ rDNA phylogenetic tree. The Lipomycetaceae form such a deep phylogenetic branch among the ascomycete yeasts that may justify their elevation to the rank of an order. The largest family of true yeasts is the Saccharomycetaceae, and its subdivision into monophyletic genera will need further molecular studies. Together with the ascospore-forming families can be classified the family Candidaceae including anamorphic forms of ascomycetous yeasts.

The basidiomycete yeasts with simple septal pore are placed in the order Sporidiales of the Urediniomycetes [10,11]. Formerly, it was related to Ustilaginales, but no phylogenetic relatedness has been demonstrated between the two groups. The related anamorphs are included in the family Sporobolomycetaceae that is no longer confined only to ballistoconidial species. Yeasts with dolipore septa are divided according to the type of basidia: Tremellales with septate basidia whereas Filobasidiales with aseptate basidia. Cryptococcaceae is the family that corresponds to anamorphs in both orders.

\section{Delineation of genera and species}

Traditionally, yeast genera were mainly differentiated by morphological criteria, particularly the type of sexual spores, whereas species were delineated using physiological features such as the fermentation and assimilation of substrates. Information provided by molecular studies has exerted the largest impact at these taxonomic levels [1]. It is now generally accepted that strains showing $70 \%$ or greater nuclear DNA homology are conspecific. The degree of nDNA relatedness usually corresponds to the ability of interfertility, on the other hand, species with high DNA relatedness may show differences in phenotypic characters. This has led to reducing many species and even genera to synonymy, e.g. the nitrate assimilating species of the former genus Hansenula has been placed among the nitrate-negative species of the genus Pichia. The DNA/DNA homology studies have also been very useful in establishing teleomorph - anamorph connections, e.g. between species of Hanseniaspora and Kloeckera as well as Dekkera and Brettanomyces.

The taxonomy of the genus Saccharomyces has undergone significant changes with the use of molecular analysis $[12,13]$. The once 41 species has been reduced to 7 and recently increased to 16 due to re-examination by molecular methods. The species currently accepted in the genus can be divided into two groups: Saccharomyces sensu stricto, and Saccharomyces sensu lato, in addition, there is an outlying species, S. kluyveri. The Saccharomyces sensu stricto complex includes four species ( $S$. cerevisiae, $S$. bayanus, S. pastorianus, and $S$. paradoxus), which have been established by DNA relatedness and are indistinguishable from each other by the conventional morphological and physiological tests. Sensitive molecular methods (karyotyping, mitochondrial DNA restriction analysis, RAPD, PCR-ribotyping) differentiate most of the Saccharomyces 
species, with the exception of the very closely related species pair, $S$. bayanus and $S$. pastorianus.

Phylogenetic analysis based on the rRNA genes and their intergenic spacer sequences $\left(\mathrm{ITS}_{1}, \mathrm{ITS}_{2}\right)$ showed that the genus Saccharomyces is heterogeneous and its species are interdispersed on the phylogenetic tree with members of related ascomycetous genera such as Torulaspora, Zygosaccharomyces and Kluyveromyces [14]. E.g. S. kluyveri forms a group, which includes two Kluyveromyces and two Zygosaccharomyces species. Clearly, the current phenotypic delineation of species and genera differs markedly from the phylogenetically related groups and awaits taxonomic restructuring.

\section{Identification and typing}

Molecular techniques offer the opportunity for rapid and reliable identification of yeasts. One way is to develop oligonucleotide probes that hybridize with species-specific sequences [15]. These so-called signature sequences have so far been determined for relatively few species, and a larger database is required for successful application of the method. More promising is the comparison of $18 \mathrm{~S}$ rDNA sequences of unknown isolates with those obtained from type strains. The latter can be retrieved from computerized databases that contain an increasing number of complete sequences. Instead of complete sequences, analysis of restriction fragments of PCR-amplified rDNA (ribotyping) is also an effective way of identification, this too, however, requires a prior database of known species.

Different molecular approaches mentioned before are sensitive enough to detect intraspecific differences and provide means to type strains that are applied in industrial production, attributed to specific ecological niches or involved in clinical cases. Polymorphisms is common for nuclear and mitochondrial DNA sequences and fragments, and can be analysed with various methods that are relatively easy to perform, accurate, reproducible and rapid. With yeasts, karyotyping of chromosomal DNA and PCR-based methods using appropriate primers have been effectively applied for biotyping and characterizing intraspecies diversity of strains $[16,17]$.

\section{Conclusions}

Molecular studies have had a great impact on the taxonomy of yeasts and over the last decade their classification has started to undergo major alterations. One of the most significant contributions has come from nDNA reassociations which have brought the precise delineation of several species, re-establishing a number of them while reducing others to synonyms. The other most significant contributions have been provided by sequencing of various rDNA regions which allowed to assess phylogenetic relationships at both lower and higher taxonomic levels. The data accumulated show already that restructuring to a large extent of current taxonomic schemes will be necessary. As a 
consequence, however, numerous nomenclatural changes are inevitable, and this is a major obstacle in constructing a phylogenetically sound classification that also provides a reliable base for the identification.

\section{REFERENCES}

1. Kurtzman,C.P.: Molecular taxonomy of the yeasts. Yeast 10, 1727-1740 (1994).

2. Deák,T.: Methods for the rapid detection and identification of yeasts in foods. Trends Food Sci Technol 6 , 287-292 (1995).

3. Kurtzman,C.P., Fell,J.W. (eds): The Yeasts, a Taxonomic Study. 4th ed., Elsevier, Amsterdam. 1998.

4. Taylor,J.W., Swann,E.C., Berbee,M.L.: Molecular evolution of ascomycete fungi: phylogeny and conflict. In Hawksworth,D.L. (ed.): Ascomycete Systematics: Problems and Perspectives in the Nineties. Plenum Press, New York. 1994. pp. 201-212.

5. Swann,E.C., Taylor,J.W.: Phylogenetic perspectives on basidiomycete systematics: evidence from the $18 \mathrm{~S}$ rRNA gene. Can J Bot 73(Suppl.), 862-868 (1995).

6. Nishida,H., Sugiyama,J.: Archiascomycetes: detection of a major new lineage within the Ascomycota. Mycoscience 35, 361-366 (1994).

7. Taylor,J.W.: Making the Deuteromycota redundant: a practical integration of mitosporic and meiosporic fungi. Can J Bot 73(Suppl.), 754-759 (1995).

8. Kurtzman,C.P., Robnett,C.J.: Orders and families of ascomycetous yeasts and yeast-like taxa compared from ribosomal RNA sequence similarities. In Hawksworth,D.L. (ed.): Ascomycete Systematics: Problems and Perspectives in the Nineties. Plenum Press, New York. 1994. pp. 249-257.

9. Kurtzman,C.P., Robnett,C.J.: Molecular relationships among hyphal ascomycetous yeasts and yeast-like taxa. Can J Bot 73(Suppl.), 824-830 (1995).

10. Boekhout,T., Fonseca,A., Campaio,J-P., Golubev,W.I.: Classification of heterobasidiomycetous yeasts: characteristics and affiliation of genera to higher taxa of basidiomycetes. Can J Microbiol 39, 276-290 (1993).

11. Swann,E.C., Taylor,J.W.: Phylogenetic diversity of yeast-producing basidiomycetes. Mycol Res 99, 12051210 (1995).

12. Barnett,J.A.: The taxonomy of the genus Saccharomyces Meyen ex Reess: a short review for nontaxonomists. Yeast 8, 1-23 (1992).

13. Deák,T., Lehoczkiné,T.J.: Classification of important Saccharomyces and related genera of yeasts in food industry based on the new results of molecular biology. (in Hungarian) Élelmezési Ipar 49, 140-146 (1995).

14. James, S.A., Cai,J., Roberts,I.N., Collins,M.D.: A phylogenetic analysis of the genus Saccharomyces based on 18S rRNA gene sequences: description of Saccharomyces kunashiriensis sp. nov. and Saccharomyces martinae sp. nov. Int J Syst Bacteriol 47, 453-460 (1997).

15. Fell,J.W.: rDNA targeted oligonucleotide primers for the identification of pathogenic yeasts in a polymerase chain reaction. J Ind Microbiol 14, 475-477 (1995).

16. Querol,A., Barrio,E., Ramon,D.: A comparative study of different methods of yeast strain characterization. Syst Appl Microbiol 15, 439-444 (1992).

17. Bostock,A., Khattak,M.N., Matthews,R., Burne,J.: Comparison of PCR fingerprinting, by random amplification of polymorphic DNA, with other molecular typing methods for Candida albicans. J Gen Microbiol 139, 2179-2184 (1993). 